\title{
Looking for two targets at the same time: One search or two?
}

\author{
CATHLEEN M. MOORE and ALLEN M. OSMAN \\ University of California, San Diego, La Jolla, California
}

\begin{abstract}
A considerable amount of evidence suggests that, under conditions of high discriminability, subjects are able to process multiple elements in a visual display simultaneously when searching for a single target among distractors. Relatively little emphasis, however, has been placed on the question of whether subjects can search for and detect multiple targets simultaneously. This latter question is the focus of the present report. In two experiments, we compare performance in single-target and multiple-target detection tasks in order to investigate whether or not multiple targets can be detected simultaneously. In Experiment 1, subjects searched for one or two targets that were defined by color. In Experiment 2, subjects searched for a color and/or a letter target. When the two targets were presented in the same location (e.g., a red X when Target 1 was red and Target 2 was an X), they seemed to be detected simultaneously. Implications for object-based processing of visual information are discussed.
\end{abstract}

Much work in the area of visual search has focused on questions concerning the spatial "mode" of search. Specifically, investigators have tried to determine under what conditions (if any) subjects are able to process all elements of a visual display simultaneously and under what conditions they must process each item in turn. The former mode is known as spatially parallel search; the latter mode is known as spatially serial search.

One method of distinguishing between spatially parallel and spatially serial search is to manipulate the number of elements in a display and observe how response time varies as a function of the number of elements present (e.g., Egeth, Jonides, \& Wall, 1972; Neisser, 1967; Treisman, Sykes, \& Gelade, 1977). Typically, a single target item is specified (e.g., an X), which subjects are to detect from among displays of distracting items. The number of distractors in the display can be varied from few to many. If each element is processed in turn, then each element that is processed before the target item is found will add to total search time. Spatially serial search therefore predicts a positive, monotonic relationship between the number of elements in the display and the time required to find the target. In contrast, if all elements are processed simultaneously, search time should be determined only by the processing of the target. Spatially parallel search therefore predicts that the time required to find the target should be unaffected by the number of elements in the display. This method has been used exten-

While conducting this research, the first author was supported by a National Science Foundation graduate fellowship. The authors are grateful to Toby Mordkoff, Charles Eriksen, Jeff Miller, Hal Pashler, Jeremy Wolfe, and two anonymous reviewers for their helpful comments on earlier drafts of this work. Correspondence should be addressed to $C$. $M$. Moore, Department of Psychology, University of Califomia, San Diego, La Jolla, CA 92093-0109 (e-mail: cmoore@ucsd.edu). sively to identify conditions under which search is spatially serial or spatially parallel (see Treisman \& Gelade, 1980, for an introduction; for objections to this approach, see Townsend, 1972, 1990).

There is, however, another way in which we may speak of parallel and serial search. Consider a situation in which subjects must search for two targets. In this case, they might conduct a single search in which they simultaneously look for both targets. Alternatively, they might conduct two searches in turn: one for the first target and another for the second. We will refer to simultaneous search for multiple targets as functionally parallel search; separate searches for each target will be called functionally serial search. Notice that the two senses of parallel and serial search - that is, functional and spatial - are logically independent of each other. A functionally serial search might consist of multiple spatially parallel searches; conversely, a single spatially serial search might consist of multiple functionally parallel searches.

Neisser (1967) developed a set of concepts that are similar to those of functionally parallel and functionally serial search. He applied the terms "operationally parallel" and "operationally sequential" to two alternative modes of processing that underlie the analysis of multiple features in his model of pattern recognition. In contrast to Neisser's terms, functionally parallel and functionally serial are here intended to describe processes that underlie search for multiple targets.

Functionally parallel and functionally serial search can be distinguished by using a method analogous to that described for spatial modes of search. Rather than observing search time as a function of the number of elements present, search time can be observed as a function of the number of targets sought. If search is functionally serial, then search time should increase with additional targets. In contrast, if search is functionally parallel, then search 
time should be no greater than the longest time required for any of the targets individually.

The present study was concerned with the question of whether or not functionally parallel search is possible. Previous research had suggested that when multiple targets are to be detected, at least a portion of the search process must be functionally serial (Quinlan \& Humphreys, 1987). We replicated and extended these findings and report conditions under which this limitation does not necessarily apply.

\section{Multiple-Target Tasks}

In order to address questions about the functional modes of search, tasks that involve multiple targets must be used. Multiple targets may be specified for search tasks in two different ways. First, subjects may be asked to make a positive response if they find any of the specified targets (e.g., " "respond 'target present' if you find either an X or an $\mathrm{O}$ in the display"). We will refer to this type of multiple-target task as an oR task. Alternatively, they may be asked to make a positive response only if they find all of the targets (e.g., "respond 'target present' only if you find both an $X$ and an $O$ "). We will refer to this second type of multiple-target search as an AND task. ${ }^{1}$ AND tasks should not be confused with conjunction-search tasks (e.g., Treisman et al., 1977). Under conjunction search, a target is defined by the spatial conjunction of multiple features. For example, subjects might be asked to indicate whether or not there is a red X present among red Os and green Xs. In this case, the correct response is not determined by two separate features; the target must be a single item that is both red and an $\mathrm{X}$. In a comparable AND task, subjects would search for something red plus an $\mathrm{X}$, regardless of whether the features were part of the same element.

When considering issues of the functional mode of search, it is useful to think of search tasks as being composed of two components: scanning of the display for the target(s) and subsequent detection of the target(s) (see Sagi \& Julesz, 1985). To scan for multiple targets simultaneously requires that, while viewing a display, subjects are able to look for more than one target at the same time. To detect multiple targets simultaneously requires that subjects are able to register more than one target as present at the same time. The terms functionally parallel and functionally serial may refer to either or both components of the search process. For search to be completely functionally parallel, both the scanning and detection components must be functionally parallel.

OR tasks and AND tasks that specify the same targets can be used to distinguish between the scanning and detection components of search. OR tasks require that subjects scan displays for multiple targets, but they need only detect a single target. AND tasks, in contrast, require that subjects both scan for multiple targets and detect the presence of multiple targets. If scanning can be functionally parallel but detection is functionally serial, then an AND task should be slower than the corresponding OR task be- cause of the time required for the additional target detection(s). As we shall see, this logic has been used to argue that target detection must be functionally serial and that, by implication, AND tasks can never be entirely functionally parallel (Quinlan \& Humphreys, 1987).

\section{Previous Research Using Multiple-Target Tasks}

OR tasks have been investigated within the context of visual search (e.g., Kaplan \& Carvellas, 1965; Neisser, Novick, \& Lazar, 1963; Treisman, 1988; Treisman \& Gelade, 1980), as well as memory scanning (e.g., Sternberg, 1969) and the development of automaticity (e.g., Schneider \& Shiffrin, 1977). AND tasks, in contrast, have been used less often (Farell, 1984; Quinlan \& Humphreys, 1987; see also Arguin \& Cavanagh, 1988).

There is evidence that $O$-task processing can be functionally parallel under some conditions, whereas under others, it seems to be functionally serial. Neisser et al. (1963) reported that subjects were no slower to scan for any of as many as 10 target letters than they were to scan for a single target letter. Similarly, Treisman (1988) found that subjects could scan for any of three different colors as quickly as they could scan for a single color. These results suggest that subjects were able to scan for multiple targets in parallel, since search time was no greater for multiple targets than it was for a single target. Under other conditions, however, scanning for multiple targets seems to be functionally serial. Treisman (1988) found that when subjects searched for any one of three targets from different stimulus dimensions, they were slower than when they searched for only one of the targets. In addition, results from studies investigating memory search have often suggested that memory sets (i.e., the set of targets that require a "positive" response) are scanned serially (e.g., Stemberg, 1969). Finally, Schneider and Shiffrin (1977) report evidence for both functionally parallel and functionally serial scanning, depending on various conditions concerning target-distractor mappings and degree of practice.

Regardless of the reason for the discrepancies, there do seem to be at least some conditions under which $O R$ tasks can be functionally parallel. This suggests that the scanning component of search can be functionally parallel. But what of the detection component? Since or tasks require only a single target detection, they cannot be used to discriminate between functionally parallel and functionally serial target detection. AND tasks, in contrast, require the detection of multiple targets as well as scanning for multiple targets.

A study by Quinlan and Humphreys (1987) suggests that detection of multiple targets occurs in series (cf. Arguin \& Cavanagh, 1988). In a set of visual search experiments, subjects searched for multiple targets in both AND and OR tasks. Search times were substantially slower in the AND tasks than in the OR tasks. The authors interpreted this slowing (hereafter referred to as AND-task slowing) as evidence that the detection component of search is functionally serial. Since the or tasks involved only a single 
target detection, whereas the AND tasks involved the detection of two targets, it was reasonable to hypothesize that AND-task slowing was caused by the additional target detection required in the AND task. ${ }^{2}$

This interpretation of AND-task slowing seems to have been influenced by a set of experiments reported by Duncan (1980) in which subjects were required to make multiple target detections. The task, in one case, was to report the number of targets that appeared among a small set of distractors. The items were presented very briefly, either simultaneously or in successive presentations of only a few at a time. Subjects were better at detecting multiple targets when they appeared in successive presentations than when they appeared simultaneously. In contrast, subjects were no better at reporting the presence of a single target when the stimuli were presented successively rather than simultaneously. These data suggested that target detection required a limited capacity mechanism whereas the processing of nontargets could be functionally parallel. Duncan's results relate to those of Quinlan and Humphreys insofar as the latter observed an advantage for OR tasks (which required only a single target detection) over AND tasks (which required multiple target detections). In accordance with Duncan's interpretation, they concluded that a serial mechanism must be invoked twice in the AND condition but only once in the OR condition, thereby causing the observed AND-task slowing.

This interpretation of AND-task slowing has an important implication-namely, AND tasks can never be entirely functionally parallel. By definition, AND tasks require subjects to detect the presence of multiple targets. Thus, subjects may be able to scan for the targets simultaneously, but, according to the above interpretation, they must detect each target in series. Therefore, at least a portion of the search process-the detection component-must be functionally serial. In everyday terms, this implies, for example, that only one face at a time can be sought and identified in a crowd or that only a single obstacle at a time can be detected while driving a car. The interpretation of AND-task slowing offered by Quinlan and Humphreys (1987), however, relies on the premise that the only effective difference between the AND and the OR tasks in their study was the detection of one versus two targets. As will be argued below, there is reason to question whether this was, in fact, the only important difference between their two tasks.

\section{Focus of the Present Research}

The purpose of the present study was to identify the source of AND-task slowing. We replicated the slowing found by Quinlan and Humphreys (1987) and further investigated its source. There were two differences between the AND and OR conditions in the original study other than the difference in the number of to-be-detected targets. It may be that multiple-target search can be functionally parallel-up to and including target detection-if one or the other of these confounds is eliminated.

Cross-dimension interference. The first alternative difference is that targets were always defined from separate stimulus dimensions (e.g., color and form). This means that subjects had not only to detect two targets in the AND tasks, they also had to detect those two targets in two different stimulus dimensions. There may be some process associated with detecting multiple pieces of information in multiple stimulus dimensions, rather than the need to detect two targets per se, that requires serial processing. Past research has shown that search times are affected by whether multiple targets are defined from different stimulus dimensions or from the same stimulus dimension (e.g., Treisman, 1988; Wolfe et al., 1990).

Cross-location interference. The second confound concerns the fact that the two targets in the AND tasks always occurred in two different locations. If, for example, the two targets were blue and C (Quinlan \& Humphreys, 1987, Experiment 1), it was never the case that a blue $\mathrm{C}$ was present. Again, it is possible that there is some process associated with detecting multiple pieces of information in multiple locations, rather than the need to detect two targets per se, that requires serial processing. The role of location as an index of selective attention has previous support (e.g., Nissen, 1985; Posner, Nissen, \& Ogden, 1978; Treisman et al., 1977).

Overview of experiments. This study was designed to determine the source of AND-task slowing and, thereby, its implications for functionally parallel versus functionally serial search. We have outlined three differences between the AND and OR tasks in which the slowing was originally observed: (1) the number of targets to be detected (one in the or tasks and two in the AND tasks), (2) the number of stimulus dimensions from which the tobe-detected targets were defined (one in the oR tasks and two in the AND tasks), and (3) the number of locations in which the targets appeared (one in the or tasks and two in the AND tasks).

In the present study, we compared single target detection in an OR task with double target detection in an AND task. In each of the experiments, we eliminated one of the remaining differences between the AND- and oR-task conditions outlined above. In Experiment 1, we defined the two targets from a single stimulus dimension in order to eliminate the possibility of cross-dimension interference. In Experiment 2, we included trials in which the two targets were presented in a single location in order to eliminate the possibility of cross-location interference. If AND-task slowing is caused by either of these two confounds rather than by the need to detect multiple targets, then the effect should not occur in one (or either) of the two experiments. ${ }^{3}$ We also included single-target search tasks in both experiments for comparison with the multipletarget tasks. This comparison allowed assessment of the functional mode of the scanning component. 


\section{GENERAL METHOD}

\section{Subjects}

Separate groups of 14 and 16 subjects from the University of California, San Diego subject pool participated in Experiments 1 and 2 , respectively. Their participation partially fulfilled a course requirement. Each subject was tested in a single 1-h session. All had normal or corrected-to-normal vision and were naive to the purpose of the experiment.

\section{Apparatus and Stimuli}

Display events and data collection were controlled by an ATcompatible microcomputer. All stimuli were presented on a VGA color monitor. Each display consisted of 6,10 , or 16 colored letters on a black background. The letters were red, green, blue, and gray Xs and Os. The specific combination of each depended on experiment, group, and condition (described below).

Each letter subtended, on average (across letter types and allowing for slightly different head positions of the subjects), $0.66^{\circ} \times$ $1.76^{\circ}$ of visual angle. The letters could appear in any of 16 locations in a $4 \times 4$ matrix. When full, the matrix covered an area of $6.15^{\circ} \times 8.3^{\circ}$ in the center of the screen. The distance from edge to edge between two laterally adjacent letters was $1.5^{\circ}$ horizontal and $0.5^{\circ}$ vertical.

Task

The task was to indicate with a buttonpress the presence or absence of the prespecified target(s) within an array of distractor elements. Responses were made with left and right index fingers on the $\mathrm{z}$ and / keys of the keyboard. Half the subjects responded "target present" with their right hand; the other half responded "target present" with their left hand. "Target absent" was indicated with the opposite hand.

\section{Design}

In both experiments, the type of search required (task type) was varied within subjects but between blocks of trials. There were four different task types in Experiment 1: an OR task, an AND task, and two single-feature tasks. There were five different task types in Experiment 2: an OR task, two AND tasks, and two single-feature tasks. More detailed descriptions of task types are given below for each experiment.

In both experiments, two additional within-subject factors were varied within blocks: display size $(6,10$, or 16 letters) and trial type (target-present, target-absent). Within a given block, each display size occurred equally often, and exactly one half of the trials of each display size were target-present trials.

\section{Procedure}

A session consisted of a series of short (10-trial) practice blocks followed by a set of longer (36-trial) experimental blocks. One practice block was included for each task type in the experiment. Thus, there were four practice blocks in Experiment 1 and five practice blocks in Experiment 2. Experiment 1 included 16 experimental blocks -4 for each of the 4 task types. Experiment 2 included 15 experimental blocks- 3 for each of the 5 task types. Order of both the practice blocks and the experimental blocks was determined randomly for each subject.

Each block began with instructions indicating the target or targets for that block. The instructions remained on the screen for 5 sec. Each trial began with a centrally presented fixation cross $(+)$. After $900 \mathrm{msec}$, the fixation cross was replaced by the task display. (Details regarding task displays are discussed separately for each experiment below.) The display disappeared upon response, and after $1,500 \mathrm{msec}$, the fixation cross of the next trial was presented.

The subjects were instructed to respond as quickly as possible during the experimental blocks and to try to keep the number of errors per block to fewer than 5 . Following an error, a tone $(800 \mathrm{~Hz})$ was sounded for $300 \mathrm{msec}$ at a comfortable volume. After each block, the subjects were shown their mean response times and number of errors for that and all previous blocks. They were advised to rest between blocks and to resume the experiment when they were ready by making a keypress.

\section{EXPERIMENT 1}

Experiment 1 tested the hypothesis that AND-task slowing is caused by cross-dimension interference. Multiple targets were defined from a single stimulus dimensioncolor. If AND-task slowing is caused by targets defined from separate stimulus dimensions, then there should be no slowing in the present AND condition.

\section{Method}

Task types. The subjects searched for red and/or green items among colored distractors. For half of the subjects, the items were always Xs; for the other half, the items were always Os. There were two multiple-target conditions: $O R$, in which subjects searched for red or green items (red and green were never simultaneously present), and Within-Dimension AND (W/I DIM AND), in which subjects searched for both red and green items. We also included two single-target conditions: Red-only and Green-only.

Displays. Displays were generated for each target-present trial by first determining a single example of the target(s) required for the relevant task condition. Distractors were then drawn from a set of potential distractors and added to the display until the required display size was met. Displays for target-absent trials were generated from the distractor set only. Table 1 summarizes the targets and distractor sets used for each task type in Experiment 1.

For the OR task, the distractors were gray and blue. Whether a given distractor was gray or blue was determined randomly for each distractor for each trial. Thus, for a given trial, it was possible to have a single blue distractor with multiple gray distractors (or a single gray distractor with multiple blue distractors). In this way, a target, when present, was not necessarily the only singleton in the display, and singleton distractors could appear on target-absent trials.

For the W/I DIM AND task, distractors were chosen for a given trial from blue, gray, and one of the two target colors (red or green). This allowed target-absent trials to contain one or the other of the target colors, but not both. Again, distractors were chosen randomly for each trial from the potential set of distractors for that trial.

Finally, for the two single-feature tasks, the distractors were chosen randomly for each trial from blue, gray, and the nontarget color (i.e., green in the case of Red-only, and red in the case of Green-only).

\section{Results}

Preliminary analyses showed that there were no systematic differences associated with the type of stimulus used ( $\mathrm{O}$ vs. $\mathrm{X}$ ) or response-hand mapping. All further

\section{Table 1}

Stimulus Conditions by Task Type in Experiment 1

\begin{tabular}{|c|c|c|}
\hline Task Type & Target & Distractor Set \\
\hline OR & Red or green & Gray and blue \\
\hline W/I DIM AND & Red and green & $\begin{array}{c}\text { Gray, blue, and red } \\
\text { or* } \\
\text { Gray, blue, and green }\end{array}$ \\
\hline $\begin{array}{l}\text { Red-only } \\
\text { Green-only }\end{array}$ & $\begin{array}{l}\text { Red } \\
\text { Green }\end{array}$ & $\begin{array}{l}\text { Gray, blue, and green } \\
\text { Gray, blue, and red }\end{array}$ \\
\hline
\end{tabular}

*Which set was used was determined randomly for each trial in the $\mathrm{w} / \mathrm{I}$ DIM AND condition. 
analyses were collapsed across these groups of subjects. The target-present trials of the or condition were separated for analyses with respect to which target was present: Or-red trials were those in which a red item was present; oR-green trials were those in which a green item was present.

Display-size analyses. Separate analyses of variance (ANOVAs) were conducted for target-present and targetabsent conditions. Both mean response times (RT) and error rates (ER) were analyzed. Target-present trials showed a significant but minimal effect of display size on RT [ 7 msec per item; $F(2,26)=8.46, p<.01]$ but not on ER $[F(2,26)=1.72, p>.19]$. There was no significant effect of display size in the target-absent trials for RT $(F<1)$. However, a $0.15 \%$ per item decrease in ER did reach significance $[F(2,26)=4.67, p<.02]$. None of the task $\times$ display size interactions were significant [RT, $F(8,104)=1.80, \mathrm{p}>.08$, for target-present trials and $F<1$ for target-absent trials; ER, $F(8,104)=$ $1.46, \mathrm{p}>.18$, for target-present trials and $F<1$ for target-absent trials]. The minimal effect of display size on RT for target-present trials, as well as the greater accuracy with larger displays on target-absent trials, suggests that search was not spatially serial overall (Treisman \& Gelade, 1980; Wolfe, Cave, \& Franzl, 1989). Moreover, since the task $\times$ display size interactions were not significant, there is little evidence that search was spatially serial within any of the individual task conditions.

Between-task comparisons. Mean RTs and ERs are shown for all conditions collapsed across display size in Table 2. Planned comparisons between task conditions showed that multiple-target search was always slower than component single-target searches. Subjects were $36 \mathrm{msec}$ faster to report the presence of a red item when looking only for red in the Red-only condition than when looking for red or green in the oR condition $[t(13)=5.36, p<$ $.01]$. They were $35 \mathrm{msec}$ faster to report the presence of green when looking only for green than when looking for red or green $[t(13)=5.45, p<.01]$. The difference between the or and single-target conditions for the targetabsent trials only approached significance $[t(13)=1.93$, $.05<p<.07$ for Red-only; $t(13)=1.94, .05<\mathrm{p}<$ .07 for Green-only].

Finally, the most interesting comparison revealed that AND-task slowing did occur in the W/I DIM AND condition. Target-present trials in the W/I DIM AND condition were slower than those in both the oR-red condition [47 msec, $t(13)=4.35, p<.01]$ and the or-green condi- tion [41 msec, $t(13)=3.06, p<.01]$. The target-absent trials showed the same pattern of results. Subjects were $73 \mathrm{msec}$ slower to report "target absent" in the w/I DIM AND condition than they were in the oR condition $[t(13)=$ $6.84, p<.001]$.

There was no evidence that the pattern of results shown by the between-task comparisons in the RT data was caused by a speed-accuracy tradeoff. The same comparisons were done with the ER data, and the only significant $(p<.05)$ trends were such that slower conditions produced greater errors.

\section{Discussion}

The results of Experiment 1 indicate that crossdimension interference cannot be the sole cause of the AND-task slowing observed by Quinlan and Humphreys (1987). In the present experiment, targets were defined from a single stimulus dimension. If the AND-task slowing was caused entirely by the need to detect two targets that were defined from two different stimulus dimensions, then we should have observed no slowing in the present experiment. The W/I DIM AND condition, however, did show an overall slowing relative to the OR condition. Therefore, AND-task slowing must have had another source.

\section{EXPERIMENT 2}

The results of Experiment 1 are consistent with the interpretation of AND-task slowing offered by Quinlan and Humphreys (1987). It may be that when searching for multiple targets in an AND task, subjects must detect each target in series. Before accepting this conclusion, however, we must also rule out between-location interference as the cause of AND-task slowing. In Experiment 1, the targets always appeared in separate locations on targetpresent AND-task trials, as they did in the experiments of Quinlan and Humphreys (1987). Thus, not only did the AND and OR conditions differ with respect to the number of targets to be detected, they also differed in terms of the number of locations in which the targets appeared. In Experiment 2, we eliminated this difference by including trials in which the two targets appeared in a single element of the display. If the AND condition is still slower than the oR condition, we can rule out cross-location interference as the sole cause of AND-task slowing.

In Experiment 2, the subjects searched for a particular color and a particular letter. Half of the subjects searched

Table 2

Mean Response Times (in Milliseconds) and Percent Error Rates

in Experiment 1

\begin{tabular}{lccccc}
\hline \multirow{2}{*}{$\begin{array}{c}\text { Task } \\
\text { Condition }\end{array}$} & \multicolumn{2}{c}{ Target-Present } & & \multicolumn{2}{c}{ Target-Absent } \\
\cline { 2 - 3 } \cline { 5 - 6 } \cline { 5 - 6 } & Response Time & Error Rate & & Response Time & Error Rate \\
\hline OR & 442 & 3.3 & & - & 450 \\
$\quad$ Red & 448 & 3.3 & & - & - \\
$\quad$ Green & 489 & 2.2 & & 523 & - \\
W/1 DIM AND & 406 & 1.9 & & 438 & 4.6 \\
Red-only & 413 & 2.2 & & 434 & 1.4 \\
Green-only & & &
\end{tabular}


for red items and/or an X; the other half searched for green items and/or an $O$. We will describe the conditions for the red and/or X group. The details for the latter group were equivalent, except that green was substituted for red, and $\mathrm{O}$ was substituted for $\mathrm{X}$.

\section{Method}

Task types. There was again an oR condition in which the subjects searched for red or $X$ (red and $X$ never appeared simultaneously). However, in Experiment 2, there were two AND conditions. The first was a Between-Locations AND (BTWN LOC AND) condition in which the two targets, when present, appeared in two different elements of the display (i.e., a green $\mathrm{X}$ and a red $\mathrm{O}$ ). The second was a within-locations AND (W/I LOC AND) condition in which the two targets, when present, appeared in the same element (i.e., a red X). Again, we included the two corresponding single-target conditions: Color-only, in which subjects searched for red only, and Form-only, in which subjects searched for $\mathrm{X}$ only.

Displays. As in Experiment 1, displays were generated for each target-present trial by first determining a single example of the target(s) required for the relevant task condition. Distractors were then drawn from a set of potential distractors and added to the display until the required display size was met. Displays for target-absent trials were generated from the distractor set only. Table 3 summarizes the targets and distractor sets used for each task type in Experiment 2.

For each of the AND conditions, distractors were randomly chosen for a given trial from among either green Os and red Os or from among green Os and green Xs. Thus, distractors could include one or the other of the two target features (red or X), but never both. For the single-target Color-only condition, distractors were randomly chosen from among green Os and green Xs. Distractors for the single-target Form-only condition were randomly chosen from among green Os and red Os.

\section{Results}

Preliminary analyses showed that there were no systematic differences associated with the type of stimulus sought (red and/or X vs. green and/or O) or responsehand mapping. All further analyses were collapsed across these groups of subjects. The target-present trials from the or condition were separated according to which target was present: oR-color for those in which the target color was present and OR-form for those in which the target letter was present.

Display-size analyses. Two-way ANOVAs (task type $X$ display size) were conducted for target-present and target-absent trials separately. Both mean RT and mean ER were analyzed. There was no significant effect of display size on either RT $[F(2,30)=1.9, p>.16$, for the target-present trials; $F<1$ for the target-absent trials] or ER $(F<1$ for both the target-present and the targetabsent trials). Moreover, none of the interactions between display size and task type attained significance for either RT $(F<1$ for both the target-present and target-absent trials) or ER [ $F<1$ for the target-present trials; $F(8,120)$ $=1.09, \mathrm{p}>.37$ for the target-absent trials]. The lack of significant main effects of display size and the lack of significant task type $\times$ display size interactions suggest that search was not spatially serial either overall or within any of the individual task conditions.

Between-task comparisons. Mean RT and ER for all task conditions, collapsed across display size, are shown in Table 4. Multiple-target conditions were, again, always slower than single-target conditions. The subjects were $102 \mathrm{msec}$ slower to report the presence of a target color in the OR-color condition than in the Color-only condition $[t(15)=5.58, p<.01]$. Similarly, they were 52 msec slower to report the presence of a target letter in the OR-form condition than in the corresponding Formonly condition $[t(15)=3.18, p<.01]$. The interaction between target component (color vs. letter) and search condition (OR vs. single feature) for the target-present trials was significant $[t(15)=2.68, p<.05]$. In the target-absent condition, the subjects were about $82 \mathrm{msec}$ faster to report the absence of the target in the Color-only condition than in the oR condition $[t(15)=5.14, p<$ $.01]$. They were also $30 \mathrm{msec}$ faster to report the absence of the target in the Form-only condition than in the or condition, but this difference was not statistically reliable $[t(15)=1.3, p>.1]$.

AND-task slowing was observed when the two targets appeared in separate locations but not when the same two targets appeared in the same location. Subjects were slower to detect two targets in the BTWN LOC AND condition than they were to detect a single target in either of the oR conditions [ $108 \mathrm{msec}$ for oR-color, $t(15)=4.56$, $p<.01 ; 86 \mathrm{msec}$ for oR-form, $t(15)=4.52, p<.01]$. The magnitude of AND-task slowing here is equivalent to that observed by Quinlan and Humphreys (1987)-about

Table 3

Stimulus Conditions by Task Type in Experiment 2*

\begin{tabular}{lll}
\hline \multicolumn{1}{c}{ Task Type } & \multicolumn{1}{c}{ Target } & \multicolumn{1}{c}{ Distractor Set } \\
\hline $\begin{array}{l}\text { OR } \\
\text { BTWN LOC AND }\end{array}$ & $\begin{array}{l}\text { Red or X } \\
\text { Red and X } \\
\text { (Red O and green X) }\end{array}$ & $\begin{array}{l}\text { Green Os } \\
\text { Green Os and red Os } \\
\text { or } \dagger\end{array}$ \\
W/I LOC AND & $\begin{array}{l}\text { Red and X } \\
\text { (Red X) }\end{array}$ & $\begin{array}{l}\text { Green Os and green Xs } \\
\text { Green Os and red Os } \\
\text { or }\end{array}$ \\
Color-only & Red & $\begin{array}{l}\text { Green Os and green Xs } \\
\text { Form-only }\end{array}$ \\
\hline
\end{tabular}

*These stimuli were those used for the group of subjects who searched for red and/or $X$. For the group who searched for green and/or $O$, substitute "green" for "red", and " $X$ " for "O." + Which set was used was determined randomly for each trial in the AND conditions. 
Table 4

Mean Response Times (in Milliseconds) and Percent Error Rates in Experiment 2

\begin{tabular}{|c|c|c|c|c|}
\hline \multirow{2}{*}{$\begin{array}{c}\text { Task } \\
\text { Condition } \\
\end{array}$} & \multicolumn{2}{|c|}{ Target-Present } & \multicolumn{2}{|c|}{ Target-Absent } \\
\hline & Response Time & Error Rate & Response Time & Error Rate \\
\hline $\mathrm{OR}_{\mathrm{R}}$ & & & 540 & 2.0 \\
\hline Color & 528 & 4.4 & - & - \\
\hline Form & 550 & 6.2 & - & - \\
\hline BTWN LOC AND & 636 & 3.7 & 604 & 4.1 \\
\hline W/I LOC AND & 544 & 2.0 & 580 & 3.0 \\
\hline Color-only & 426 & 3.1 & 458 & 1.3 \\
\hline Form-only & 498 & 1.7 & 510 & 0.9 \\
\hline
\end{tabular}

100 msec. In contrast, the subjects were not significantly slower to detect the two targets in the W/I LOC AND condition than they were to report either of the targets in the OR-color and OR-form conditions $(t<1$ for both $O R$ color and $O R$ form).

The extent to which performance in the W/I LOC AND condition was similar to that in the OR conditions can be seen in Figure 1. Shown here are the Vincentized cumulative reaction-time distributions for the target-present trials in the multiple-target conditions of Experiment $2{ }^{4}$ As can be seen, the BTWN LOC AND condition was slower than both of the or conditions across the entire distribution. In contrast, the distribution for the W/I LOC AND condition is almost indistinguishable from that of the slower of the two or conditions.

Finally, target-absent times were slower in both the BTWN LOC AND and the W/I LOC AND condition, than they were in the oR condition [64 msec, $t(15)=6.42, p<$ .01 , for BTWN LOC AND; $40 \mathrm{msec}, t(15)=2.86, p<$ .05 , for W/1 LOC AND].

The same between-task comparisons revealed several significant effects in the error data for both target-present and target-absent trials. Most of these effects were such that more errors were made in the slower conditions. However, the subjects did make more errors when detecting single targets in the or conditions than they did when detecting both targets in the $\mathrm{W} / \mathrm{I}$ LOC AND condition $[t(15)=2.69, p<.02$, for OR-color; $t(15)=3.95, p<$ .01 , for OR-form]. It is unlikely, however, that a speed-accuracy tradeoff obscured differences in RT between the W/I LOC AND and the OR-form conditions. The almost identical RT distributions produced by these two conditions would require almost perfect compensation for RT differences at all percentiles.

\section{Discussion}

The results of Experiment 2 indicate that cross-location interference is a major cause of AND-task slowing. Detecting targets in the BTWN LOC AND condition, in which the two targets appeared in two different locations, was considerably slower than detecting either target in the oR condition. However, when the same two targets appeared in a single location, the subjects were no slower to detect both targets than they were to detect either target in the OR condition (see Pollatsek \& Digman, 1977, for results similar to those from the BTWN LOC AND and W/I LOC AND conditions). Experiment 2 therefore provides an example of conditions under which multiple targets were detected in an AND task as quickly as a single target was detected in an OR task. This establishes the possibility that detection can be functionally parallel and, by implication, that AND tasks can be entirely functionally parallel.

\section{GENERAL DISCUSSION}

This study was concerned with whether search for multiple targets can ever be functionally parallel. It was noted that for multiple-target search to be entirely functionally parallel, subjects must both scan for all of the targets simultaneously and detect all of the targets simultaneously. Previous research had provided conditions under which scanning for multiple targets seemed to be functionally parallel (e.g., Neisser et al., 1963; Treisman, 1988). However, Quinlan and Humphreys (1987) found that subjects were slower to respond to the presence of both of two targets in an AND task than they were to respond to a single target in an OR task. Since AND and oR tasks are the same with respect to the number of targets scanned

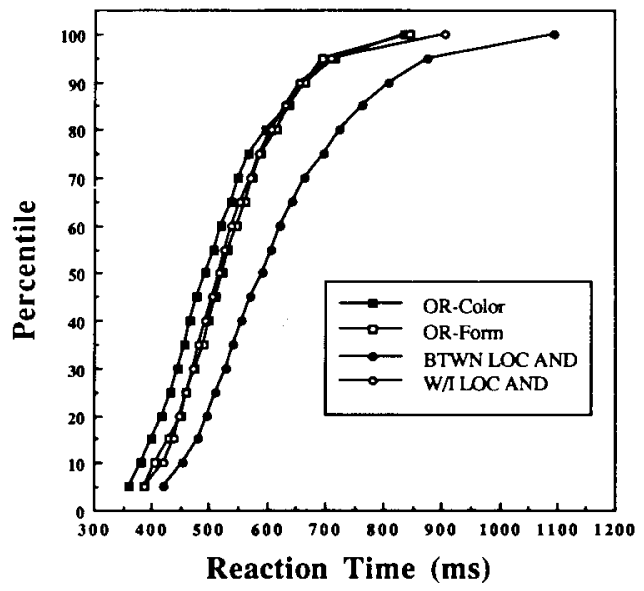

Figure 1. Vincentized cumulative distribution functions for reaction times in each of the multiple-target, target-present conditions of Experiment 2. The target-present trials of the oR condition are separated with respect to which target was present: OR-color for when the target color was present and OR-form for when the target letter was present. 
for, but differ with respect to the number of target detections, it was concluded that AND-task slowing was caused by a functionally serial detection component. This interpretation implies that AND tasks can never be entirely functionally parallel since, by definition, they require the detection of multiple targets. In two experiments, we investigated alternative explanations of AND-task slowing.

Experiment 1 showed that AND-task slowing occurred even when the two targets were defined from the same stimulus dimension. The data from Experiment 1 allowed us to rule out the hypothesis that the slowing observed by Quinlan and Humphreys (1987) was caused entirely by cross-dimension interference. Experiment 2, however, showed that when the two targets were presented in a single item (e.g., red and X presented as a red X), the AND condition was no longer slower than the OR condition. Thus, Experiment 2 provides conditions under which subjects were no slower to detect multiple targets than they were to detect a single target.

An important aspect of the results, however, is that scanning for multiple targets appears to have been functionally serial in the present study. Searching for multiple targets in the or task was slower than searching for single targets in the component single-target tasks in both Experiments 1 and 2. There are several possible reasons why the structure of the present study may have led the subjects to scan for multiple targets in series. First, specific features that served as targets on some trials appeared as part of the distractors on other trials. Moreover, since our subjects participated in only a single 1-h session, they were not highly practiced with any of the tasks. It has been suggested that under conditions such as these, subjects engage in functionally serial scanning (Schneider \& Shiffrin, 1977). Further slowing of the oR task in Experiment 2 may have been caused by the targets having been defined from separate stimulus dimensions. The slowing of the OR task relative to the single-target tasks was about twice as great in Experiment 2 as it was in Experiment 1, in which the targets were defined from a single stimulus dimension. Support for this later hypothesis has been found in a study in which features were consistently mapped to targets or distractors. Under these conditions, Treisman (1988) observed that OR-task search times were slower than single-target search times only when the targets were defined from separate stimulus dimensions.

Although serial scanning may at first appear inconsistent with parallel detection, the scanning mode does not logically constrain the detection mode. For example, it is possible for subjects to scan for multiple targets in series and, on completion, to detect those targets in parallel. We cannot make the strong claim that AND-task processing was entirely functionally parallel in the present study, since scanning may have been functionally serial. We can, however, conclude that functionally parallel detection is not ruled out by the AND-task slowing observed by Quinlan and Humphreys (1987). Moreover, since there are conditions under which scanning has been found to be functionally parallel, we can further suggest, contrary to the original implication of AND-task slowing, that it is possible that AND tasks can be entirely functionally parallel, both in the scanning and the detection components.

\section{Implications for Models of Visual Search}

Two implications for models of visual search can be derived from the present research. The first is that, insofar as models of search are intended to accommodate all search situations-including those involving multiple targets-it may be useful to consider a distinction between a scanning component and a detection component of search processes (Sagi \& Julesz, 1985). Whether scanning is functionally parallel or serial is in principle independent of whether detection is functionally parallel or serial. Therefore, when a search involves multiple targets, scanning and detection must be considered separately. Any general model of visual search, then, should allow for this distinction.

Some models of visual search, those which do not explicitly address functional modes of search, already include an implicit distinction between scanning and detection. Models in which processing is thought to proceed from one object to another, such that search order is determined by characteristics of the display, imply separate scanning and detection processes (e.g., Duncan \& Humphreys, 1989; Egeth, Virzi, \& Garbart, 1984; Hoffman, 1978, 1979; Treisman \& Sato, 1990; Wolfe et al., 1989). The scanning component determines the search order; the detection component is the process of analyzing the separate elements.

A second implication concerns the influence of location on search processes. Models that maintain that location information is used only when feature conjunction is required cannot readily account for the W/I LOC AND condition being faster than the BTWN LOC AND condition in the present study (Experiment 2). For example, one version of the feature integration theory (FIT) presented by Treisman (1985) maintains that feature search (i.e., search that does not require features to be conjoined) is mediated by activity in feature maps that contain no readily available information about the location of the items in which those features appeared. The targets in both the BTWN LOC AND and the W/I LOC AND conditions were single features (e.g., red and $\mathrm{X}$ ) that did not require conjunction. Therefore, according to this model, the targets should have been detected by the same means in the two conditions, that is, without reference to location information. However, the relative locations of the two features did affect search time. The two features were detected more quickly when they appeared in the same location than when they appeared in different locations.

Other versions of FIT (e.g., Treisman, 1988; Treisman \& Souther, 1985) posit that attention is drawn to the location of target items with salient features, thus resulting in other features of those elements being accessed. In this case, one would predict that the targets would be detected more quickly in the W/ $\mathrm{I}$ LOC AND task than in the BTWN LOC AND task. If, however, it takes any time to ac- 
cess the additional features once attention has been drawn to an item, then the W/I LOC AND condition should have been slower than the or condition, which was not the case. In any event, the present results provide further evidence that models of visual search require some mechanism of detection-such as a process which can draw attention to the location of salient features-beyond simple access of a given feature map (see also Johnston \& Pashler, 1990).

\section{Processing of Multi-Dimensional Stimuli}

An issue similar to what we have been calling functionally parallel versus functionally serial processing has been addressed before in tasks other than visual search. There have been a number of studies reported that were concerned with the question of whether the processing of separable attributes (e.g., shape and size) of a single stimulus can be processed simultaneously or must be processed serially (e.g., Biederman \& Checkosky, 1970; Downing \& Gossman, 1970; Egeth, 1966; Hawkins, 1969; Lindsay \& Lindsay, 1966; Nickerson, 1967; Saraga \& Shallice, 1973). The tasks in these earlier studies were usually comparison tasks wherein a subject reported whether multiattribute items were the same as, or different from, a memorized target item. Items could differ in terms of a single attribute or many attributes. Most investigators concluded that multiple attributes could be processed simultaneously (but see Egeth, 1966, and Lindsay \& Lindsay, 1966).

The present study suggests that these conclusions may be limited to conditions under which the attributes are parts of the same stimulus. In the earlier multidimensional studies, the to-be-processed attributes were presented as parts of a single stimulus. Here, we report results that are consistent with simultaneous processing of multiple attributes when those attributes were parts of the same stimulus (W/I LOC AND condition, Experiment 2). We also report results, however, that are consistent with serial processing of the same attributes when those attributes were parts of different stimuli (BTWN LOC AND condition, Experiment 2). The question of whether or not multiple attributes are, or can be, processed simultaneously may depend critically on whether the attributes are from a single item or whether they are from separate items.

\section{Object-Based Selective Processing}

Why should simultaneous processing of multiple targets occur only when the targets are presented in the same location? There is a growing body of literature that suggests that attentional mechanisms-such as those presumably employed in visual search-can be driven by objectbased parameters (Baylis \& Driver, 1992; Kahneman \& Henik, 1981; Kahneman, Treisman, \& Gibbs, 1992; see Kanwisher \& Driver, 1992, for a review). In the present study, when two pieces of information were presented in the same location, they were also presented in the same object. Therefore, the elimination of AND-task slowing in the W/I LOC AND condition of Experiment 2 may have been due to the two targets having appeared within the same object rather than in the same location.
The results of the present study fit well with the hypothesis that there is a serial detection mechanism that is limited to detecting a single object at a time rather than being limited to detecting a single target at a time. The serial-target-detection interpretation of AND-task slowing (Quinlan \& Humphreys, 1987) was motivated in part by Duncan's (1980) results, which demonstrated an advantage for successively presented targets over simultaneously presented targets. On the basis of these results, Duncan hypothesized that a detection mechanism must be invoked for each target in turn. In a later study, however, he found that when the response was based on two features that appeared in a single object, performance was equivalent to that when the response was based on a single target feature (Duncan, 1984; see also Treisman, Kahneman, \& Burkell, 1983, and Lappin, 1967). In Experiment 2 of the present study, targets were contained within a single object in the OR and the W/I LOC AND tasks, but they appeared in separate objects in the BTWN LOC AND condition. Since AND-task slowing was observed in the BTWN LOC AND condition but not in the W/I LOC AND condition, it is reasonable to hypothesize that the serial detection mechanism may be limited to the processing of a single object at a time rather than a single target.

\section{Conclusions}

This study demonstrates three main points. First, the distinction between a scanning component and a detection component can be important when considering functional modes of search. Second, functionally parallel search for multiple targets-both scanning and detection-need not be ruled out by the results reported by Quinlan and Humphreys (1987). Finally, if multipletarget search can be entirely functionally parallel, then one of the necessary conditions is likely to be that the two targets appear in the same location/object.

\section{REFERENCES}

Arguin, M., \& Cavanagh, P. (1988). Parallel processing of two disjunctive targets. Perception \& Psychophysics, 44, 22-30.

BAYLIS, C. G., \& DrIVER, J. (1992). Visual parsing and response competition: The effect of grouping factors. Perception \& Psychophysics, 51, 145-162.

Biederman, I., \& Checkosky, S. F. (1970). Processing redundant information. Journal of Experimental Psychology, 83, 486-490.

Downing, B. D., \& Gossman, J. R. (1970). Parallel processing of multidimensional stimuli. Perception \& Psychophysics, 8, 57-60.

DunCAN, J. (1980). The locus of interference in the perception of simultaneous stimuli. Psychological Review, 87, 272-300.

DunCaN, J. (1984). Selective attention and the organization of visual information. Journal of Experimental Psychology: General, 113, 501-517.

DunCan, J., \&umphreys, G. W. (1989). Visual search and stimulus similarity. Psychological Review, 96, 433-458.

Egeth, H. E. (1966). Parallel versus serial processes in multidimensional stimulus discrimination. Perception \& Psychophysics, 1 , 245-252.

Egeth, H. E., Jonides, J., \& Wall, S. (1972). Parallel processing in complex visual discrimination. Cognitive Psychology, 3, 674-698.

Egeth, H. E., VIRZI, R. A., \& GarbarT, H. (1984). Searching for conjunctively defined targets. Journal of Experimental Psychology: Human Perception \& Performance, 10, 32-39. 
FARELL, B. (1984). Attention in the processing of complex visual displays: Detecting features and their combinations. Joumal of Experimental Psychology: Human Perception \& Performance, 10, 40-64.

Hawkins, H. L. (1969). Parallel processing in complex visual discrimination. Perception \& Psychophysics, 5, 56-64.

Hoffman, J. E. (1978). Search through a sequentially presented visual display. Perception \& Psychophysics, 23, 1-11.

Hoffman, J. E. (1979). A two-stage model of visual search. Perception \& Psychophysics, 25, 319-327.

Johnston, J. C., \& PASHLER, H. (1990). Close binding of identity and location in feature perception. Journal of Experimental Psychology: Human Perception \& Performance, 16, 843-856.

Kahneman, D., \& Henik, A. (1981). Perceptual organization and attention. In M. Kubovy \& J. R. Pomerantz (Eds.), Perceptual organization (pp. 181-211) Hillsdale, NJ: Erlbaum.

Kahneman, D., Treisman, A., \&ibbs, B. J. (1992). The reviewing of object files: Object-specific integration of information. Cognitive Psychology, 24, 178-211.

KANWISHer, N., \& Driver, J. (1992). Objects, attributes and visual attention: Which, what and where. Current Directions in Psycholog ical Science, 1, 1-5.

Kaplan, I. T., \& Carvellas, T. (1965). Scanning for multiple targets. Perceptual \& Motor Skills, 21, 239-243.

LAPPIN, J. (1967). Attention in the identification of stimuli in complex visual displays. Journal of Experimental Psychology, 75, 321-328.

Lindsay, R. K., \& LiNDSAY, J. M. (1966). Reaction time and serial versus parallel information processing. Joumal of Experimental Psychology, 71, 294-303.

NeIsSER, U. (1967). Cognitive psychology. New York: AppletonCentury-Crofts.

Neisser, U., Novick, R., \& Lazar, R. (1963). Searching for ten targets simultaneously. Perceptual \& Motor Skills, 17, 955-961.

NiCKERSON, R. S. (1967). "Same" - "Different"' response times with multiattribute stimulus differences. Perceptual \& Motor Skills, 24, 543-554.

NisSEN, M. J. (1985). Assessing features and objects: Is location special? In M. I. Posner \& O. S. M. Marin (Eds.), Attention and performance $X I$ (pp. 205-219). Hillsdale, NJ: Erlbaum.

Pollatsek, A., \& Digman, L. (1977). Dependent spatial channels in visual processing. Cognitive Psychology, 9, 326-352.

Posner, M. I., Nissen, M. J., \& OGden, W. C. (1978). Attended and unattended processing modes: The role of set for spatial location. In H. L. Pick \& I. J. Saltzman (Eds.), Modes of perceiving and processing information (pp. 137-147). Hillsdale, NJ: Erlbaum.

Quinlan, P. T., \& HumpHreys, G. W. (1987). Visual search for targets defined by combinations of color, shape, and size: An examination of the task constraints on feature and conjunction searches. Perception \& Psychophysics, 41, 455-472.

SAGI, D., \& JULESZ, B. (1985). Detection versus discrimination of visual orientation. Perception, 14, 619-628.

Saraga, E., \& Shallice, T. (1973). Parallel processing of the attributes of single stimuli. Perception \& Psychophysics, 13, 261-270.

SCHNEIDER, W., \& SHIFFrIN, R. M. (1977). Controlled and automatic human information processing: Detection, search, and attention. Psychological Review, 84, 1-66.

STERNBerg, S. (1969). Memory scanning: Mental processes revealed by reaction time experiments. American Scientist, 57, 421-457.

Thомas, E. A. C., \& Ross, B. H. (1980). On appropriate procedures for combining probability distributions within the same family. Journal of Mathematical Psychology, 21, 136-152.

TownSEND, J. T. (1972). Some results concerning the identifiability of parallel and serial processes. British Joumal of Statistical Psychology, 25, 168-199.

TowNSEND, J. T. (1990). Serial vs. parallel processing: Sometimes they look like Tweedledum and Tweedledee, but they can (and should) be distinguished. Psychological Science, 1, 46-54.
Treisman, A. (1985). Preattentive processing in vision. Computer Vision, Graphics, and Image Processing, 31, 156-177.

Treisman, A. (1988). Features and objects: The fourteenth Bartlett memorial lecture. Quarterly Journal of Experimental Psychology, 40A, 201-237.

Treisman, A. M., \& Gelade, G. (1980). A feature-integration theory of attention. Cognitive Psychology, 12, 97-136.

Treisman, A., Kahneman, D., \& Burkell, J. (1983). Perceptual objects and the cost of filtering. Perception \& Psychophysics, 33 527-532.

Treisman, A., \& Sato, S. (1990). Conjunction search revisited. Journal of Experimental Psychology: Human Perception \& Performance, 16, 459-478

Treisman, A., \&outher, J. (1985). Search Asymmetry: A diagnostic for preattentive processing of separable features. Journal of $E x-$ perimental Psychology: General, 114, 285-310.

Treisman, A., Sykes, M., \& Gelade, G. (1977). Selective attention and stimulus integration. In S. Dornic (Ed.), Attention and performance $V I$ (pp. 333-361). Hillsdale, NJ: Erlbaum.

Wolfe, J. M., Cave, K. R. \& Franzl, S. L. (1989). Guided search: An alternative to the feature integration model for visual search. Jour nal of Experimental Psychology: Human Perception \& Performance, $15,419-433$

Wolfe, J. M., Yu, K. P., Stewart, M. I., Shorter, A. D., Friedman-Hill, S. R., \& CAVE, K. R. (1990). Limitations on the parallel guidance of visual search: Color $\times$ color and orientation $\times$ orientation conjunctions. Journal of Experimental Psychology: Human Perception \& Performance, 16, 879-892.

\section{NOTES}

1. AND tasks have also been called both tasks when only two targets have been specified (e.g., Quinlan \& Humphreys, 1987). We use the term "AND task" here to emphasize the generality of the concept to all multiple target detections.

2. Farell (1984) reported results from an experiment in which subjects were slower to report the presence of either of two targets in an OR task than they were to report the presence of both targets in an AND task. The difference between these results and those of Quinlan and Humphreys (1987) may be attributable to differences in the displays used in the two studies. In contrast to the many-item displays used by Quinlan and Humphreys, Farell's displays included only two items. This meant that there were only two possible target-present displays in the AND condition but many possible target-present displays in the OR condition. Perhaps subjects were able to memorize the particular targetpresent displays in the AND condition but had to search for the targets in the oR condition. We report here a replication of the Quinlan and Humphreys (1987) result under search conditions similar to theirs in which there were many elements in the displays.

3. It is, in fact, possible that the slowing was caused by the combination of different stimulus dimensions and different locations. However, defining an AND task in which the targets are both defined from a single stimulus dimension and can both be presented in the same location requires a judgment call on what constitutes the same location or the same stimulus dimension. For example, a green disk surrounded by a red annulus could be interpreted as either one location or two. Similarly, a single disk varying in saturation and brightness could be interpreted as either one dimension or two. Advocates of any position could argue their point, regardless of the data, on the basis of the chosen definitions of dimension and location. We therefore did not include this condition.

4. Vincentizing entails averaging the reaction times of the individual subjects at each of the quantiles shown along the ordinate (Thomas \& Ross, 1980).

(Manuscript received April 24, 1992; revision accepted for publication September 9, 1992.) 